\title{
Orbital Angular Momentum in Scalar Diquark Model and QED
}

Hikmat $\mathrm{BC}^{1}$

The University of Texas at El Paso, USA

Email: hbbc@utep.edu,

Matthias Burkardt

New Mexico State University, USA

Email: burkardt@nmsu.edu

We compare the orbital angular momentum of the 'quark' in the scalar diquark model as well as that of the electron in QED (to order $\alpha$ ) obtained from the Jaffe-Manohar decomposition to that obtained from the Ji relation. We estimate the importance of the vector potential in the definition of orbital angular momentum.

\footnotetext{
${ }^{1}$ Presented by Hikmat BC at LIGHTCONE 2011, 23 - 27 May, 2011, Dallas.
} 


\section{Introduction}

The question of how the total angular momentum $\frac{1}{2}$ of the proton is contributed by the spins of the quarks and gluons and their orbital angular momenta, has been a puzzle since the European Muon Collaboration (EMC) announced its result in late 80s. Very little contribution to the proton's spin was found from the spin of the quarks, and hence so- called proton "spin crisis" has existed[1, 2]. After almost 20 years of vigorous theoretical and experimental effort, only about $30 \%$ of the proton spin is contributed by spin of the quarks. Researchers are actively engaged in to the quest for the remaining $70 \%$ of the proton's spin. It appeared more clear that this rest of the spin of the proton should be contributed by the orbital angular momentum (OAM) of the quarks and gluons and the polarization of the gluons. Recently, there are many debates on the proper way of decomposing the total spin of the proton into the OAM and spin contribution from quarks and gluons

\subsection{Ji Spin Sum Rule}

Ji proposed a decomposition of the $\vec{z}$ - component of the angular momentum of the nucleon

$$
\frac{1}{2}=\frac{1}{2} \sum_{q} \Delta q+\sum_{q} L_{q}^{z}+J_{g}^{z}
$$

whose terms are matrix elements of the corresponding terms of the $0 x y$ - components of the following angular momentum tensor

$$
M^{0 x y}=\frac{1}{2} \sum_{q} q^{\dagger} \Sigma^{z} q+\sum_{q} q^{\dagger}(\vec{r} \times i \vec{D})^{z} q+[\vec{r} \times(\vec{E} \times \vec{B})]^{z}
$$

where $i \vec{D}=i \vec{\partial}-g \vec{A}$. In this decomposition, each term can be expressed as the expectation value of a manifestly gauge invariant local operator. Also the total angular momentum of the quark can be expressed in terms of the generalized parton distributions(GPDs) as

$$
\begin{aligned}
J_{q}^{z} & =\frac{1}{2} \Delta q+L_{q}^{z} \\
& =\frac{1}{2} \int_{0}^{1} d x x\left[q(x)+E_{q}(x, 0,0)\right]
\end{aligned}
$$

which can be measured in deeply virtual Compton scattering(DVCS) or calculated in lattice gauge theory [3, 4, 5, 6].

\subsection{Jaffe - Manohar Spin Sum Rule}

Jaffe and Manohar proposed a decomposition of the $\vec{z}$ - component of the angular momentum of the nucleon at the light - cone frame as

$$
\frac{1}{2}=\frac{1}{2} \sum_{q} \Delta q+\sum_{q} \mathscr{L}_{q}^{z}+\frac{1}{2} \Delta G+\mathscr{L}_{g}^{z}
$$

where these terms are defined as matrix elements of the corresponding terms in the $+x y$ component of the angular momentum tensor 


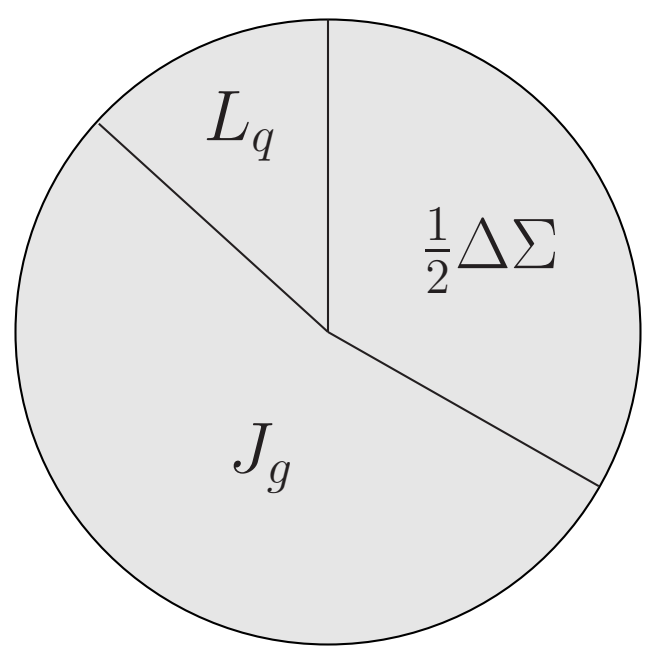

Figure 1: Ji decomposition

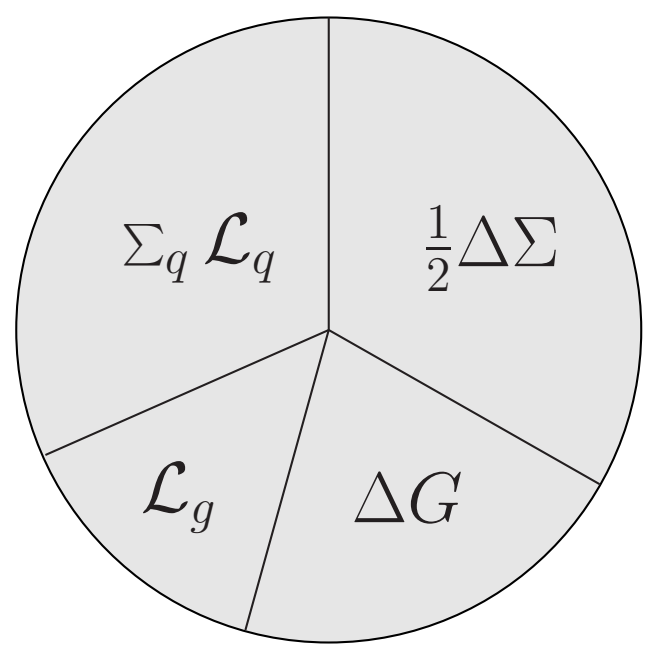

Figure 2: Jaffe- Manohar decomposition

$$
M^{+x y}=\frac{1}{2} \sum_{q} q_{+}^{\dagger} \gamma_{5} q_{+}+\sum_{q} q_{+}^{\dagger}(\vec{r} \times i \vec{\partial})^{z} q_{+}+\varepsilon^{+-i j} \operatorname{TrF}^{+i} A^{j}+2 \operatorname{Tr} F^{+j}(\vec{r} \times i \vec{\partial})^{z} A^{j}
$$

where $q_{+}=\frac{1}{2} \gamma^{-} \gamma^{+} q$ is the dynamical component of the quark field operators and $A^{+} \equiv$ $A^{0}+A^{+}=0$ is the light cone gauge.

In (4) and (5), the first and third terms are the 'intrinsic' contributions to the angular momentum $J^{z}=+\frac{1}{2}$ of the nucleon and can be interpreted as spin of the quark and gluon respectively and the second and third terms are interpreted as the corresponding orbital angular momenta(OAM). The quark spin term is manifestly gauge invariant. Gluon spin is accessible experimentally and hence it is also gauge invariant. It is defined through a non - local operator in the gauges other than light- cone gauge [3, 2, 6].

The total OAM of both quark and gluon, which is gauge invariant, can be written as

$$
\mathscr{L}^{z}=\sum_{q} \mathscr{L}_{q}^{z}+\mathscr{L}_{g}^{z}=\frac{1}{2}-\frac{1}{2} \sum_{q} \Delta q-\frac{1}{2} \Delta G
$$

The expectation value of $\bar{q} \gamma^{z} \Sigma^{z} q$ vanishes for a parity eigenstate. So, one can substitute $q^{\dagger} \Sigma^{z} q \rightarrow \bar{q} \gamma^{+} \Sigma^{z} q=q_{+}^{\dagger} \gamma_{5} q_{+}$, i.e. the $\Delta q$ are same in both decompositions. All the other terms are different from each other since they are not defined through matrix elements of the same operator and one should not expect them to have the same numerical value [3, 6].

We have OAM term from Ji relation,

$$
q^{\dagger}(\vec{r} \times i \vec{D})^{z} q=\bar{q} \gamma^{0}(\vec{r} \times i \vec{D})^{z} q \rightarrow \bar{q}\left(\gamma^{0}+\gamma^{z}\right)(\vec{r} \times i \vec{D})^{z} q+q_{+}^{\dagger}(\vec{r} \times i \vec{D})^{z} q_{+}
$$

Note that the expectation value is taken in a parity eigenstate. Even in light- cone gauge, $\mathscr{L}^{z}$ and $L^{z}$ differ by the expectation value of $q_{+}^{\dagger}(\vec{r} \times g \vec{A}) q_{+}$since Eq. (7) contains the transverse component of the vector potential through the gauge covariant derivative. 


\section{Orbital Angular Momentum (OAM) in Scalar Diquark Model}

With the center of momentum and relative $\perp$ coordinates, for a two particle system[3],

$$
\begin{aligned}
\mathbf{P}_{\perp} & \equiv \mathbf{p}_{1 \perp}+\mathbf{p}_{2 \perp} \\
\mathbf{R}_{\perp} & \equiv x_{1} \mathbf{r}_{1 \perp}+x_{2} \mathbf{r}_{2 \perp}=x \mathbf{r}_{1 \perp}+(1-x) \mathbf{r}_{2 \perp} \\
\mathbf{k}_{\perp} & \equiv x_{2} \mathbf{p}_{1 \perp}-x_{1} \mathbf{p}_{2 \perp}=(1-x) \mathbf{p}_{1 \perp}-x \mathbf{p}_{2 \perp} \\
\mathbf{r}_{\perp} & \equiv \mathbf{r}_{1 \perp}-\mathbf{r}_{2 \perp}
\end{aligned}
$$

where $x_{1}=x$ and $x_{2}=1-x$ are the momentum transfer carried by active quark and the spectator respectively. One can replace the OAM operator for particle 1 by $(1-x)$ times the relative OAM in a state with $\mathbf{P}_{\perp}=0$ which gives us $\mathbf{p}_{1 \perp}=-\mathbf{p}_{2 \perp}=\mathbf{k}_{\perp}$.

$$
\mathscr{L}_{1}^{z}=\mathbf{r}_{1 \perp} \times \mathbf{p}_{1 \perp}=\left[\mathbf{R}_{\perp}+(1-x) \mathbf{r}_{\perp}\right] \times \mathbf{k}_{\perp} \rightarrow(1-x) \mathbf{r}_{\perp} \times \mathbf{k}_{\perp}=(1-x) \mathscr{L}^{z}
$$

Similarly, one can write $\mathscr{L}_{2}^{z}=x \mathscr{L}^{z}$ for particle 2 .

To compute the OAM of the quark in Ji and Jaffe and Manohar decomposition, we have the light -cone Fock state wave functions in Scalar di quark model[3, 7, 6],

$$
\begin{gathered}
\Psi_{+\frac{1}{2}}^{\uparrow}\left(x, \overrightarrow{k_{\perp}}\right)=\left(M+\frac{m}{x}\right) \psi\left(x, \mathbf{k}_{\perp}^{2}\right), \\
\Psi_{-\frac{1}{2}}^{\uparrow}\left(x, \overrightarrow{k_{\perp}}\right)=-\frac{\left(k^{1}+i k^{2}\right)}{x} \psi\left(x, \mathbf{k}_{\perp}^{2}\right) \\
|\psi|^{2}=\frac{g^{2} x^{2}(1-x)}{\left[M^{2} x^{2}-\left(M^{2}+m^{2}-\lambda^{2}\right) x+\left(\overrightarrow{k_{\perp}}+m^{2}\right)\right]^{2}}
\end{gathered}
$$

where $g$ is the Yukawa coupling and $M, m$, and $\lambda$ are the masses of the nucleon, quark and diquark respectively. Here $x$ is the momentum fraction carried by the quark and the relative momentum $\mathbf{k}_{\perp} \equiv \mathbf{k}_{e \perp}-\mathbf{k}_{\gamma \perp}$. The $\uparrow$, upper index, of the wave function represents the helicity of the nucleon and the lower index that of the quark.

According to Jaffe - Manohar decomposition, the OAM of the quark is [3]

$$
\mathscr{L}_{q}^{z}=\frac{g^{2}}{16 \pi^{3}} \int_{0}^{1} d x \int d^{2} \overrightarrow{k_{\perp}}(1-x)\left|\Psi_{\frac{-1}{2}}^{\uparrow}\right|^{2}
$$

Similarly according to Ji decomposition,

$$
\begin{gathered}
L_{q}^{z}=J_{q}^{z}-\left\langle S_{q}^{z}\right\rangle \\
J_{q}^{z}=\frac{1}{2}\left(A_{q}(0)+B_{q}(0)\right)=\frac{1}{2} \int_{0}^{1} d x x\left[q(x)+E_{q}(x, 0,0)\right] \\
A_{q}(0)=1-\frac{1}{16 \pi^{3}} \iint d x d^{2} k_{\perp}(1-x)\left[\left|\Psi_{+\frac{1}{2}}^{\uparrow}\right|^{2}+\left|\Psi_{-\frac{1}{2}}^{\uparrow}\right|^{2}\right] \\
B_{q}(0)=\frac{1}{16 \pi^{3}} \iint d x d^{2} k_{\perp} \frac{2 M(1-x)(m+M x)}{x}|\psi|^{2}
\end{gathered}
$$




$$
\left\langle S_{q}^{z}\right\rangle=\frac{1}{2}+\frac{1}{16 \pi^{3}} \iint d x d^{2} k_{\perp}\left(\frac{1}{2}\left[+1\left|\Psi_{+\frac{1}{2}}^{\uparrow}\right|^{2}-1\left|\Psi_{-\frac{1}{2}}^{\uparrow}\right|^{2}\right]-\frac{1}{2}\left[+1\left|\Psi_{+\frac{1}{2}}^{\uparrow}\right|^{2}+1\left|\Psi_{-\frac{1}{2}}^{\uparrow}\right|^{2}\right]\right)
$$

We used manifestly Lorentz invariant Pauli- Villars regularization (subtraction with heavy scalar $\lambda^{2} \rightarrow \Lambda^{2}$ ) to compute some of the divergent $\mathbf{k}_{\perp}$ integrals. Computing above integrals we found that , in scalar diquark model,

$$
L_{q}^{z}=\mathscr{L}_{q}^{z}
$$

It is not so surprising for scalar diquark model since it is not a gauge theory i.e the OAM term does not contain a gauge field term. However, the $x$-distribution of the OAM, $\left(L_{q}^{z}(x)\right.$ and $\left.\mathscr{L}_{q}^{z}(x)\right)$, are not exactly some as shown in the Figure 3

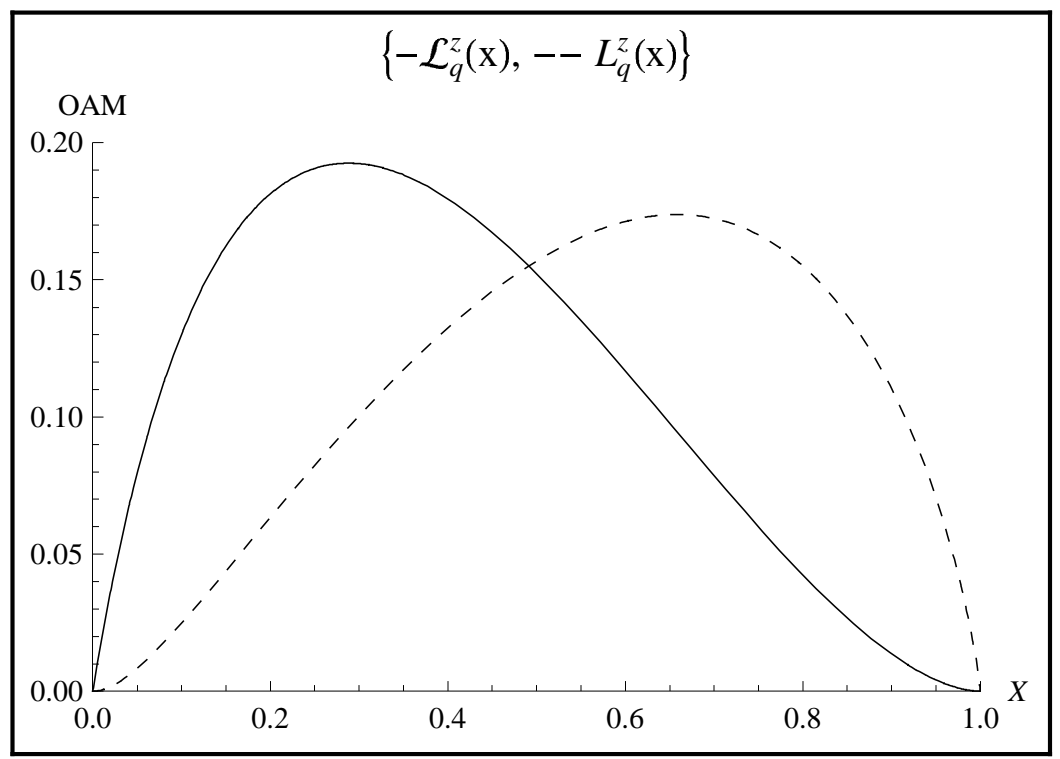

Figure 3: $x$ dependence of OAM for Ji (dashed) and Jaffe - Manohar (solid) in the scalar diquark model for parameters $\Lambda^{2}=10 \lambda^{2}=10 \mathrm{~m}^{2}$. Both in units of $\frac{g^{2}}{16 \pi^{2}}$.

\section{Orbital Angular Momentum (OAM) in QED}

The light -cone Fock state wave functions in QED perturbative theory are [7, 3, 6, 8]. There are four polarization states in the $e \gamma$ Fock component :

$$
\begin{gathered}
\Psi_{+\frac{1}{2}+1}^{\uparrow}\left(x, \overrightarrow{k_{\perp}}\right)=-\sqrt{2} \frac{\left(-k^{1}+i k^{2}\right)}{x(1-x)} \psi\left(x,{\overrightarrow{k_{\perp}}}^{2}\right), \\
\Psi_{+\frac{1}{2}-1}^{\uparrow}\left(x, \overrightarrow{k_{\perp}}\right)=-\sqrt{2} \frac{\left(+k^{1}+i k^{2}\right)}{(1-x)} \psi\left(x,{\overrightarrow{k_{\perp}}}^{2}\right), \\
\Psi_{-\frac{1}{2}+1}^{\uparrow}\left(x, \overrightarrow{k_{\perp}}\right)=-\sqrt{2}\left(m-\frac{m}{x}\right) \psi\left(x,{\overrightarrow{k_{\perp}}}^{2}\right),
\end{gathered}
$$




$$
\Psi_{-\frac{1}{2}-1}^{\uparrow}\left(x, \overrightarrow{k_{\perp}}\right)=0
$$

where

$$
\psi\left(x,{\overrightarrow{k_{\perp}}}^{2}\right)=\frac{e / \sqrt{1-x}}{m^{2}-\left({\overrightarrow{k_{\perp}}}^{2}+m^{2}\right) / x-\left({\overrightarrow{k_{\perp}}}^{2}+\lambda^{2}\right) /(1-x)}
$$

where $x$ is the momentum fraction carried by the electron and $(1-x)$ is that for the photon. $\vec{k}_{\perp}$ is the transverse component of momentum of the electron; $m$ and $\lambda$ are the masses of the electron and photon respectively.

According to Jaffe- Manohar decomposition, OAM of the electron,

$$
\mathscr{L}_{e}^{z}=\frac{1}{16 \pi^{3}} \int_{0}^{1} d x \int d^{2} k_{\perp}(1-x)\left[-1\left|\Psi_{+\frac{1}{2}+1}^{\uparrow}\right|^{2}+1\left|\Psi_{+\frac{1}{2}-1}^{\uparrow}\right|^{2}\right]
$$

Similarly, according to Ji decomposition, OAM of the electron is

$$
\begin{gathered}
L_{e}^{z}=\frac{1}{2}\left[A_{q}(0)+B_{q}(0)\right]-\left\langle S_{q}\right\rangle \\
A_{q}(0)=1+\frac{1}{16 \pi^{3}} \iint d x d^{2} k_{\perp} x\left[+1\left|\Psi_{+\frac{1}{2}+1}^{\uparrow}\right|^{2}+1\left|\Psi_{+\frac{1}{2}-1}^{\uparrow}\right|^{2}+1\left|\Psi_{-\frac{1}{2}+1}^{\uparrow}\right|^{2}\right] \\
-\frac{1}{16 \pi^{3}} \iint d x d^{2} k_{\perp}\left[+1\left|\Psi_{+\frac{1}{2}+1}^{\uparrow}\right|^{2}+1\left|\Psi_{+\frac{1}{2}-1}^{\uparrow}\right|^{2}+1\left|\Psi_{-\frac{1}{2}+1}^{\uparrow}\right|^{2}\right] \\
B_{q}(0)=\left.4 M \frac{1}{16 \pi^{3}} \iint d x d^{2} k_{\perp}(m-m x) \psi\right|^{2}
\end{gathered}
$$

Now, expectation value of spin angular momentum of the electron $\left\langle S_{q}\right\rangle$ is

$$
\begin{aligned}
\left\langle S_{q}\right\rangle= & \frac{1}{16 \pi^{3}} \iint d x d^{2} k_{\perp} \frac{1}{2}\left[+1\left|\Psi_{+\frac{1}{2}+1}^{\uparrow}\right|^{2}+1\left|\Psi_{+\frac{1}{2}-1}^{\uparrow}\right|^{2}-1\left|\Psi_{-\frac{1}{2}+1}^{\uparrow}\right|^{2}\right] \\
& +\frac{1}{2}-\frac{1}{16 \pi^{3}} \iint d x d^{2} k_{\perp} \frac{1}{2}\left[+1\left|\Psi_{+\frac{1}{2}+1}^{\uparrow}\right|^{2}+1\left|\Psi_{+\frac{1}{2}-1}^{\uparrow}\right|^{2}+1\left|\Psi_{-\frac{1}{2}+1}^{\uparrow}\right|^{2}\right]
\end{aligned}
$$

We used manifestly Lorentz invariant Pauli- Villars regularization (subtraction with heavy scalar $\lambda^{2} \rightarrow \Lambda^{2}$ ) to compute some of the divergent $\mathbf{k}_{\perp}$ integrals. Computing above integrals we found that [3, 6],

$$
\mathscr{L}_{e}^{z}=-\frac{\alpha}{2 \pi} \int_{0}^{1} d x\left(1-x^{2}\right) \log \frac{(1-x)^{2} m^{2}+x \Lambda^{2}}{(1-x)^{2} m^{2}+x \lambda^{2}} \stackrel{\Lambda \rightarrow \infty, \lambda \rightarrow 0-}{\longrightarrow} \frac{\alpha}{4 \pi}\left[\frac{4}{3} \log \frac{\Lambda^{2}}{m^{2}}-\frac{2}{9}\right]
$$

and

$$
\begin{aligned}
L_{e}^{z}= & -\frac{\alpha}{4 \pi} \int_{0}^{1} d x\left(1+x^{2}\right)\left[\log \frac{(1-x)^{2} m^{2}+x \Lambda^{2}}{(1-x)^{2} m^{2}+x \lambda^{2}}-\frac{(1-x)^{2} m^{2}}{(1-x)^{2} m^{2}+x \lambda^{2}}-\right. \\
& \left.\frac{(1-x)^{2} m^{2}}{(1-x)^{2} m^{2}+x \Lambda^{2}}\right]
\end{aligned}
$$




$$
\stackrel{\Lambda \rightarrow \infty, \lambda \rightarrow 0}{\longrightarrow}-\frac{\alpha}{4 \pi}\left[\frac{4}{3} \log \frac{\Lambda^{2}}{m^{2}}+\frac{7}{9}\right]
$$

As long as $\Lambda^{2}>\lambda^{2}$, both the $\mathscr{L}_{e}^{z}$ and $L_{e}^{z}$ are negative regardless of the value of $\Lambda^{2}$. So, the difference between two OAMs [3] is

$$
\mathscr{L}_{e}^{z}-L_{e}^{z} \stackrel{(\Lambda \rightarrow \infty, \lambda \rightarrow 0)}{\longrightarrow} \frac{\alpha}{4 \pi}
$$

The cutoff dependence of OAMs from Ji and Jaffe - Manohar decompositions in QED is shown in Figure 4

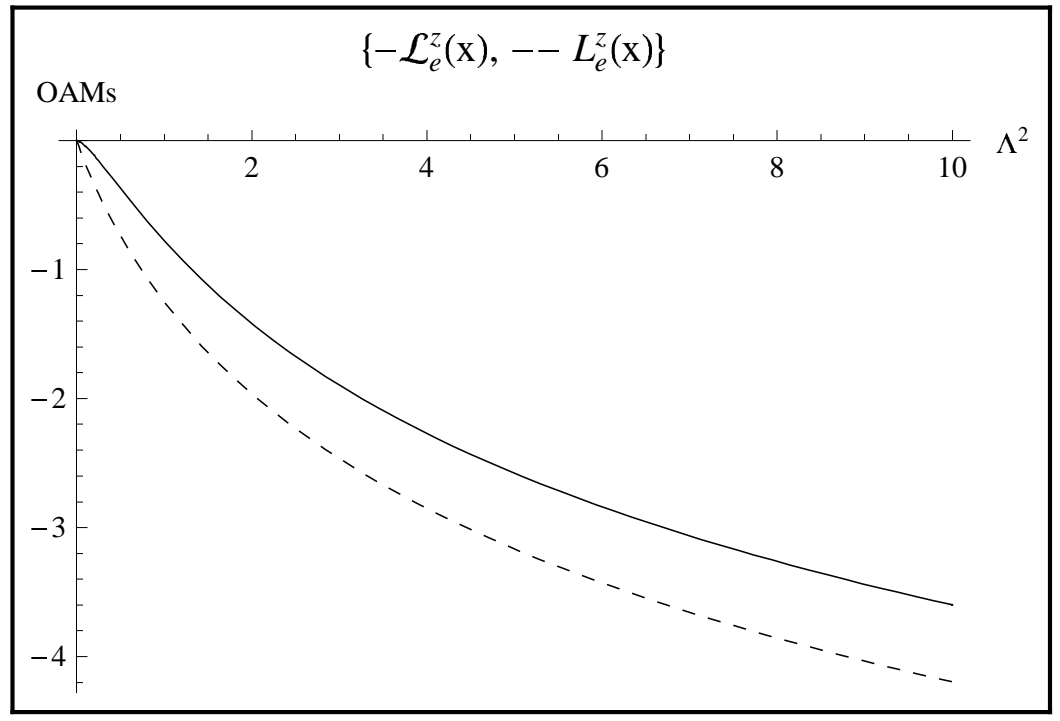

Figure 4: Cutoff dependence of OAMs: Ji (dashed) and Jaffe - Manohar (solid). Both in units of $\frac{\alpha}{4 \pi}$.

Above results allow us to evaluate the difference between two OAMs for a massive quark [3] with $J^{z}=+\frac{1}{2}$,

$$
\mathscr{L}_{q}^{z}-L_{q}^{z}=\frac{\alpha_{s}}{3 \pi}
$$

In $\mathrm{QCD}$, gluon OAM $\mathscr{L}_{g}^{z}$ is not experimentally accessible but the gluon spin is. The total angular momentum of the gluon $J_{g}^{z}$ in the Ji relation is accessible either directly by calculating gluon GPDs on a lattice and / or deeply virtual $J / \psi$ production or indirectly by subtraction $J_{g}^{z}=\frac{1}{2}-J_{q}^{z}$. One can think of calculating OAM of gluon by subtracting $\frac{1}{2} \Delta G$ in Jaffe - Manohar decomposition from $J_{g}^{z}$ in Ji decomposition but subtracting Eq. (4) from Eq. (1) gives us [3],

$$
J_{g}^{z}-\frac{1}{2} \Delta G=\mathscr{L}_{g}^{z}+\sum_{q}\left(\mathscr{L}_{q}^{z}-L_{q}^{z}\right) .
$$

i.e numerically $J_{g}^{z}-\frac{1}{2} \Delta G$ differs from $\mathscr{L}_{g}^{z}$ by the same amount as $\sum_{q} \mathscr{L}_{q}^{z}$ differs from $\sum_{q} L_{q}^{z}$. In QED, the photon spin contribution in Ji decomposition is given by 


$$
\Delta \gamma=\int_{0}^{1} d x \int \frac{d^{2} \overrightarrow{k_{\perp}}}{16 \pi^{3}}\left[\left|\Psi_{+\frac{1}{2},+1}^{\uparrow}\right|^{2}-\left|\Psi_{+\frac{1}{2},-1}^{\uparrow}\right|^{2}+\left|\Psi_{-\frac{1}{2},+1}^{\uparrow}\right|^{2}\right]
$$

For $(\Lambda \rightarrow \infty, \lambda \rightarrow 0)$, we get,

$$
J_{\gamma}^{z}-\frac{1}{2} \Delta \gamma=\mathscr{L}_{\gamma}^{z}+\frac{\alpha}{4 \pi}
$$

\section{Summary and Discussion}

In this work, we studied the angular momentum decomposition in scalar diquark model as well as that in QED as proposed by Jaffe - Manohar and that according to Ji relation [6]. Moreover, we compare OAMs of an electron in QED and that for an active quark in scalar diquark model both in Jaffe- Manohar and Ji decompositions. In the scalar diquark model, as anticipated, both the OAMs for the fermions are same but not in QED. It can be concluded that the presence of vector potential in the manifestly gauge invariant local operator for the OAM does indeed contribute significantly to the numerical value of the OAM. The differences seem

to be small which are of the order of $\frac{\alpha}{4 \pi}$ but one should accept that the fact that all the OAMs are of the order of $\alpha$. In QCD, for $\alpha_{s} \approx 0.5$ about $10 \%$ of the spin budget for the massive quark is also contributed by vector potential term [3].

\section{References}

[1] F. Myhrer and A. W. Thomas, J. Phys. G37, 023101 (2010).

[2] R. L. Jaffe and A. Manohar, Nucl. Phys. B337, 509 (1990).

[3] M. Burkardt and H. BC, Phys. Rev. D79, 071501 (2009).

[4] X.-D. Ji, Phys. Rev. Lett. 78, 610 (1997).

[5] X.-d. Ji, Nucl. Phys. Proc. Suppl. 119, 41 (2003).

[6] M. Burkardt, H. BC, and A. Jarrah, (2010).

[7] S. J. Brodsky, D. S. Hwang, B.-Q. Ma, and I. Schmidt, Nucl. Phys. B593, 311 (2001).

[8] S. J. Brodsky and S. Drell, Phys.Rev. D22, 2236 (1980). 